\title{
Essais
}

Revue interdisciplinaire d'Humanités

Hors-série 7 | 2022

Récits d'outre-thèse

\section{Cheminement dans le monde scolastique : de la fascination à la désillusion}

Journey into the scholastic world: From fascination to disillusionment

Hélène Bourdeloie

\section{(2) OpenEdition}

Journals

Édition électronique

URL : https://journals.openedition.org/essais/10783

DOI : 10.4000/essais. 10783

ISSN : 2276-0970

Éditeur

École doctorale Montaigne Humanités

Édition imprimée

Date de publication : 15 avril 2022

ISBN : 979-10-970024-00-0

ISSN : $2417-4211$

Référence électronique

Hélène Bourdeloie, «Cheminement dans le monde scolastique : de la fascination à la désillusion », Essais [En ligne], Hors-série 7 | 2022, mis en ligne le 01 février 2022, consulté le 17 janvier 2023. URL http://journals.openedition.org/essais/10783; DOI : https://doi.org/10.4000/essais.10783

Ce document a été généré automatiquement le 17 janvier 2023.

Tous droits réservés 


\title{
Cheminement dans le monde scolastique : de la fascination à la désillusion
}

\author{
Journey into the scholastic world: From fascination to disillusionment
}

\author{
Hélène Bourdeloie
}

1 C'est à l'École Normale Supérieure (ENS) de Fontenay Saint-Cloud, ensuite délocalisée à Lyon, que je fus officiellement inscrite en thèse de doctorat en sciences de l'information et de la communication et eus le désagrément d'expérimenter de plein fouet la «violence symbolique» qu'implique la fréquentation de ce type d'institution, notamment pour quelqu'un qui ne fait pas partie du sérail ${ }^{1}$. En raison de mon milieu social et de ce que je percevais de mon niveau intellectuel, je ne m'y sentais pas à ma place. D'ailleurs, non-normalienne, je n'y étais pas comme me le rappela un jour une future collègue : «Ah d'accord! Mais donc tu n'es pas normalienne en fait ! ». J'avais en effet bénéficié d'une entrée à l'école doctorale qui accueillait nouvellement des doctorant·e's externes. La suite de mon parcours eut raison de ma prétention à une place à laquelle je n'étais manifestement pas destinée.

2 Je rejoignis finalement les bancs de l'université Paris II où je soutins une thèse sur les usages du multimédia d'art et de musée et les pratiques culturelles en 2007 ; sujet qui s'inscrivait dans une histoire personnelle. Mon habitus clivé ${ }^{2}$ m'avait en effet très tôt exposée au sens de la légitimité culturelle, c'est-à-dire au principe selon lequel un degré de légitimité était associé aux pratiques culturelles qui, comme les classes sociales, étaient hiérarchisées ${ }^{3}$. Selon ce modèle, la transmission culturelle se faisait par la famille ${ }^{4}$. Mes parents ne m'avaient pas transmis les codes de la culture consacrée (peinture, sculpture, littérature, théâtre...) et le fait d'avoir un jour vu mon père, technicien non diplômé peu familier de ladite, parcourir un cédérom de Paul Cézanne sur son ordinateur, m'avait interrogée. Je l'avais vu frayer avec la peinture via un ordinateur, «outil masculin». D'une part, cette expérience me réconcilia avec la technique, en tout cas avec l'ordinateur, que j'avais toujours associé aux hommes. De 
l'autre, elle me fit penser que la hiérarchie culturelle n'était peut-être pas immuable et que la culture élitaire pouvait s'appréhender de différentes manières. Cette anecdote est assez symptomatique de l'intérêt que j'éprouvai ensuite pour les questions de genre et de numérique et de la façon dont ma trajectoire personnelle affecta mon rapport à mes objets de recherche et vice versa ${ }^{5}$.

Dans ce récit, je souhaite particulièrement interroger ma trajectoire académique dans son rapport à la science en tant que champ et pratique. Après avoir évoqué dans un premier temps les fragilités qui ont altéré mon parcours de doctorante, j'expliquerai de quelle façon, une fois mon intégration assurée dans le monde universitaire, mon rapport à la science s'est transformé, pour finir sur une réflexion sur les enjeux qui la traversent et dont j'ai fait les frais.

\section{Un parcours doctoral en demi-teinte}

$4 \mathrm{Au}$ début de ma thèse de doctorat, je ne disposais pas vraiment des armes méthodologiques ad hoc pour affronter mon objet de recherche (les usages du multimédia d'art et de musée et les pratiques culturelles) et conduire une enquête de terrain. Mon DEA (média \& multimédia) n'avait effectivement pas été satisfaisant, ni sur le plan théorique, ni méthodologique. Ainsi pris-je l'initiative de participer à des séminaires ouverts à l'EHESS (École des Hautes études en Sciences Sociales) à Paris, puis de m'inscrire à son DEA de sociologie en tant qu'auditrice libre (1999-2000). Ces enseignements opérèrent une transformation aussi bien scientifique que personnelle. J'eus la conviction qu'ils me mettaient à l'abri de rapports de domination subis ailleurs et m'aidaient à me réaliser et trouver ma voie. J'étais enfin en mesure de caractériser des dominations préalablement éprouvées : de la part de ma filiation bourgeoise qui se jouait de mon autre parenté plus populaire, ou de la part d'institutions comme l'ENS où j'essuyai quelques camouflets. Je me souviens par exemple d'un séminaire où je demandai à l'intervenant l'orthographe du nom d'un auteur et où je fus rappelée à l'ordre pour avoir outrepassé les codes en vigueur: une telle question n'avait pas sa place dans cette institution.

Après mon court passage à l'ENS en raison d'une restructuration de laboratoires expérience qui eut pour vertu de m'éclairer sur les enjeux de pouvoir qui traversaient le champ académique - je rejoignis l'Institut Français de Presse à l'université Paris II (2000), hélas à une époque où les doctorante's en sciences de l'information et de la communication, rattachée's à l'école doctorale de droit public, disposaient de faibles moyens de recherche et manquaient de visibilité. Dans ce contexte d'isolement scientifique pendant mes premières années de doctorat, je joignis des réseaux de doctorante's et contribuai, avec des acolytes, à créer une association pour doctorant·es en sciences de l'information et de la communication. Après avoir cumulé quelques emplois alimentaires, je m'investis ensuite dans l'enseignement au titre de chargée de cours puis d'ATER (attachée temporaire d'enseignement et de recherche). Arrivée à la phase de rédaction de ma thèse, je m'envolai subséquemment pour Le Caire pour y suivre mon conjoint qui y accomplissait un volontariat international en tant que chargé de presse et de communication à l'ambassade de France, poste qu'il interrompit prématurément suite à la réussite d'un concours. Pour ma part, je décidai de rester en Égypte pour y terminer ma thèse lorsqu'une intuition me souffla qu'il m'incombait - en cas de défaite universitaire -, de préparer une option subsidiaire à ma possible carrière 
de chercheure. J'avais en effet connu plusieurs proches qui, le doctorat en poche ou presque, avaient souffert d'avoir manqué une profession universitaire. Un doctorat pouvait devenir un handicap sur le marché de l'emploi. Je devais donc sécuriser mon avenir. Forte de certains atouts - je connaissais l'arabe égyptien et l'Égypte - je présentai donc, avec succès, ma candidature au poste de volontaire international qu'avait quitté mon conjoint. Je dus ainsi me vouer, pendant deux années, à mon activité professionnelle à l'ambassade tout en rédigeant mon mémoire de thèse ; deux rôles difficiles à tenir.

Durant ces dernières années de doctorat, mon quotidien m'écarta des manifestations scientifiques et réseaux de recherche français. Ma distance avec le monde universitaire et mon immersion dans une société étrangère me confrontèrent à des difficultés croissantes. Menant une double activité de doctorante et de professionnelle de la presse et de la communication, je me sentais prise dans un étau: je préparais une carrière d'enseignante-chercheure que je n'exercerais peut-être jamais et supportais, par pragmatisme, les routines d'un quotidien professionnel qui émoussait ma joie de vivre. Enfin, mon doctorat en poche, j'accusai alors les conséquences de mon isolement géographique : tandis que je commençai en France les campagnes de recrutement de maître de conférences, j'eus vent d'interrogations sur les motifs de mon séjour à l'étranger. Lors d'une audition, on me demanda les raisons de mon apprentissage de l'arabe... Il m'était parfois difficile de mesurer la nature de l'intérêt - intellectuel, scientifique ou personnel... - de certaines de ces questions et je me sentais, selon les cas, - atypique, inclassable, voire étrangère - dans le sens où l'écrit Tahar Ben Jelloun ${ }^{6}$.

7 Échouant, bien que de peu, à la première campagne d'auditions des maîtres'esses de conférences pour laquelle je m'étais déplacée à dessein depuis Le Caire, j'eus le sentiment - tromperie ou illusion - de payer mon absence. J'étais peut-être par trop déconnectée des codes académiques français. Il me fut par exemple rapporté après une audition que mon affublement avait été jugé inapproprié.

Après avoir dispensé des cours de communication à l'université Aïn Shams dans la métropole égyptienne, je dus finalement rentrer en France puis trouver un emploi. Mon expérience à l'ambassade de France en Égypte s'avéra une aubaine puisqu'elle me permit de décrocher un poste de responsable adjointe du pôle multimédia au ministère des Affaires étrangères. Pourtant, bien que valorisant et pécuniairement avantageux, ce poste, que je trouvais trop administratif, ne me satisfaisait pas. Je souhaitais me dédier à la recherche, faire du terrain et avoir la liberté de penser sans être captive d'éléments de langage. Or, ces desiderata échappaient à la fonction que j'occupais et qu'il me fallait quitter sous peine de m'éloigner de la recherche. Au bout de quelques mois, l'offre d'un post-doctorat sur l'écriture numérique à l'Université de Technologie de Compiègne (UTC) permit de nouveau mon incorporation dans le monde universitaire : il sonna le glas de mon parcours de professionnelle de la communication. Sauvée d'un naufrage dans lequel je me sentais sombrer, je revins à mes amours scientifiques. Ma réinsertion fut promptement entérinée puisque je réussis immédiatement le concours de la seconde campagne de MCF, en 2010.

\section{Mon rapport à la science : le grand désenchantement}

Étudiante puis doctorante, mon idée de la science était fort candide. Tributaire du fantasme de la pureté bachelardienne ${ }^{7}$, j'embrassai une attitude plutôt positiviste. Le 
monde scientifique me fascinait car il incarnait ce que le monde ordinaire n'était pas ; les intérêts des scientifiques me semblaient franchement nobles et leur écriture ésotérique souvent un signe de génie. Ces convictions, qui me poursuivirent amplement, m'enserrèrent dans une conception neutre de la science qui se doubla d'une attitude dénuée d'audace, consistant à dissimuler constamment ma réflexion sous le couvert d'auteur'e's et à leur tirer ma révérence. Au cours de ma thèse, plusieurs lectures m'avaient semblé impérieuses pour marquer la coupure épistémologique entre le fait scientifique et le sens commun ${ }^{8}$. J'avais lu Gaston Bachelard et parcouru Le Métier de sociologue $e^{9}$ lectures qui me semblaient impérieuses pour marquer la coupure épistémologique entre le monde ordinaire et celui de la science ${ }^{10}$. Dans le même temps, elles me condamnèrent à adopter une vision plutôt scientiste et à plaider pour le principe de neutralité axiologique propre à Max Weber ${ }^{11}$. Pourtant, je découvris plus tard, avec honte, que je n'avais originairement pas saisi ce concept qui ne signifiait pas que le ou la scientifique devait s'interdire tout jugement de valeur puisqu'il ou elle était pris dans le monde qu'il ou elle observait, mais qu'il convenait de maîtriser son rapport aux valeurs et de distinguer l'engagement de la propagande dans un contexte politique d'influence du marxisme ${ }^{12}$.

10 Une rencontre avec des investigations de terrain et des travaux en épistémologie des sciences ravisa toutefois mon rapport au champ scientifique et mon positionnement. Dans la continuité de la réflexion engagée dans ma thèse sur le désenclavement de la culture cultivée par le biais du numérique, une enquête de terrain sur Wikipédia ${ }^{13}$ interrogeant l'éclectisme culturel et le rapport à la production des savoirs m'invita à sortir d'une double impasse : ma sujétion au pouvoir de la science et mon manque d'assurance. Cette recherche affecta mon sens critique. J'eus de moins en moins foi dans l'ordre culturel bourdieusien. Pire, j'osais mettre en doute la prétendue inconditionnalité de la valeur de savoirs scientifiques; nouvelles croyances qui firent l'effet d'une cure scientifique et forgèrent mon sens critique sur la production de la science. À cet égard, l'affaire Sokal - du nom de ce physicien qui publia en 1996 un texte dit scientifique dans la revue Social Text ${ }^{14}$ - m'avait vraiment ébranlée. Ce canular, qui visait à dénoncer le manque de rigueur de la revue et la corruption éthique traversant le champ des sciences sociales, engendra une vraie querelle entre sciences. Cette affaire, dont j'eus connaissance postérieurement, ne faisait certes que conforter des travaux d'épistémologue comme Bruno Latour ${ }^{15}$ qui m'avaient donné un avant-goût des rapports de force, alliances et compromis qui peuplaient les sciences et mettaient à mal le principe d'objectivité. Sur un plan plus personnel, j'avais par ailleurs eu l'occasion d'observer les comportements de scientifiques qui, comme le racontait David Lodge dans sa littérature, illustraient les dérives du monde universitaire; ou encore des collègues qui cultivaient le mandarinat quand d'autres, dominés par leur position hiérarchique - voire de genre ou de classe - en faisaient les frais. Ces expériences théoriques et empiriques m'exhortèrent à démythifier puis à démystifier l'essence de la science et à me libérer d'inhibitions incorporées: je me sentis plus légitime dans l'exercice de ma pratique scientifique. 


\section{La science en exercices : enjeux de pouvoir et reconnaissance symbolique}

11 Le champ universitaire m'exposa très vite à certaines violences en matière d'égotisme, de sexisme ou de compétitivité. Ce que je vécus comme des violences symboliques devait peut-être à mon identité de genre et à mon origine sociale, mais aussi à des dispositions mal acquises lors de ma vie de doctorante. Ma formation doctorale ne m'avait effectivement pas initiée aux codes, valeurs ou normes encadrant la recherche en sciences sociales. Jeune maitresse de conférences, j'ignorais par exemple la règle selon laquelle, à contribution égale, la liste des auteurees devait suivre un ordre alphabétique. Lors d'une première contribution à quatre mains, j'avais ainsi, par pure courtoisie, mentionné en premier le patronyme d'une consœur qui arrivait pourtant après le mien dans l'ordre alphabétique. Un ami s'étonna de cet ordre au regard d'une égale contribution et m'expliqua le sens de cet ordre. J'en perçus plus tard les enjeux après avoir eu vent de tensions concernant la signature d'articles qui, fruit de traditions, compromis et enjeux de pouvoir ${ }^{16}$, faisait l'objet de débats constants sur la question de l'auctorialité, c'est-à-dire le lien entre l'auteure et le texte produit. Puis j'eus l'infortune d'en être victime ${ }^{17}$ dans le cadre d'un travail où, bien que mon apport du moins valorisant intellectuellement, il n'en était pas moins incontournable pour cette production pour laquelle je n'étais pourtant jamais citée.

Avec l'expérience, je compris que ce problème s'avérait pesant en sciences humaines et sociales, particulièrement sensibles à la paternité de l'écrit ${ }^{18}$. L'application déloyale de certaines normes engendrait de sérieux préjudices puisque tout l'accomplissement du travail du chercheur ou de la chercheure en souffrait en termes de visibilité et de politiques de citations. Or la reconnaissance symbolique des paire's est essentielle dans ce métier doté d'une faible valeur économique ${ }^{19}$. La question de l'auctorialité et de l'ordre des signatures s'avère d'autant plus sensible dans un contexte où la reconnaissance académique se fonde de plus en plus sur le nombre de publications signées, mode d'évaluation privilégié dans le cadre du renforcement d'une culture évaluatrice tous azimuts ${ }^{20}$. Or cette culture se développait ces dernières années par le biais d'instances comme l'AERES (Agence de l'Évaluation de la Recherche et de l'Enseignement Supérieur ${ }^{21}$ ), l'ANR (Agence Nationale de la Recherche, créées en 2006) mais aussi de mesures (loi relative aux libertés et responsabilités des universités du 10 juillet 2007, Loi de programmation pour la recherche 2021 à 2030) profondément ancrées dans la norme du marché international du classement ${ }^{22}$. Il fallait s'y résoudre. Dans la droite ligne des politiques déjà engagées dans le service public en matière de déréglementation et de "nouvelle gestion publique $»^{23}$, et plus largement de politiques européennes, la recherche et la connaissance faisaient désormais l'objet d'un management qui encourageait une «culture du résultat » aux dépens d'autres savoirs ${ }^{24}$. Face à cette pression croissante, les communautés universitaires étaient confrontées à des injonctions contradictoires: produire toujours plus (y compris en termes administratifs) en ayant de moins en moins le temps de chercher ${ }^{25}$. Tentée's de céder à ce tourbillon de la rentabilité, les universitaires risquaient alors de se livrer à une course à la bibliométrie et de sacrifier la qualité sur l'autel de la quantitée ${ }^{6}$, voire de s'adonner à des pratiques déloyales ${ }^{27}$. Ces mécanismes s'enracinaient au reste dans une tendance sociétale qui faisait l'apologie du culte de la performance. En témoignaient ces réseaux sociaux dits académiques (ResearchGate, Academia), qui sommaient les 
chercheure's d'y diffuser leurs publications sous peine d'être exclu'e's du jeu scientifique. Sur un plan plus personnel, les conséquences pouvaient être dévastatrices puisqu'elles concouraient à fabriquer une norme de l'auto-expression et de la mise en valeur de soi qui n'était pas sans engendrer de la recherche d'autopromotion et une escalade du narcissisme ${ }^{28}$. Favorisant l'effet Matthieu lorsqu'elles concentraient les pouvoirs des porteurs ou porteuses de projets qui pourtant, comptaient sur plusieurs ressources humaines, elles contribuaient à renforcer la culture de la compétition et à exacerber les tensions dans les équipes de recherche. Il s'ensuivait un "cynisme désabusé » où tout le monde perdait « à ce jeu biaisé " $^{29}$.

Je pense avoir subi les conséquences de cette idéologie managériale ${ }^{30}$ en étant accusée de plagiat. Si d'un point de vue technique, il fut relativement aisé de prouver ma bonne foi puisque j'avais conservé les traces de nos échanges et que les universités s'équipaient désormais de logiciels anti-plagiat, je n'en fus pas moins affectivement dévastée. Des affres de cette expérience, je tirai surtout des enseignements éthiques et compris que nos universités et instances françaises montraient là des limites. J'appris à cette occasion l'émersion de la fonction de "référent·e à l'intégrité scientifique » qui avait pris forme dans le cadre de la charte de déontologie des métiers de la recherche lancée par le CNRS (Centre National de Recherche Scientifique) de 2015 $5^{31}$. À l'heure où je contactai les intéressée's, le dispositif d'intégrité scientifique s'avérait néanmoins encore branlant ${ }^{32}$.En réalité, la souffrance ou victimisation - semblablement liée à une affaire de reconnaissance symbolique ${ }^{33}$ - semblait jouer des deux côtés. Dans ce contexte, l'un des référents à l'intégrité scientifique me suggéra d'abuser du «namedropping " à la faveur de mon collègue. Si j'en compris l'enjeu en termes de sensibilité, j'en perçus aussi les travers dans ma pratique scientifique car faire usage de références non lues risquait d'entraver ma liberté de raisonnement. En définitive, je me pliai néanmoins au jeu pour apaiser la situation.

Un autre effet concomitant, me semble-t-il, des effets pervers du management de la recherche concerne cette fois une enquête dont j'avais la responsabilité et qui portait sur le rôle du numérique dans le processus de deuil. Dans ce cadre, une entrevue entre ma collaboratrice stagiaire - sociologue de formation avec une expérience de terrain et une endeuillée, s'était mal déroulée, ce dont ma collaboratrice m'avait expressément fait part a posteriori. L'endeuillée menaça après l'entrevue le responsable en titre du projet de "dénoncer » auprès des milieux professionnels du funéraire nos pratiques et le «piège » de cette entrevue ${ }^{34}$. Décontenancée, je m'étais d'abord tournée vers le service de la recherche de l'université portant le projet, puis vers son service juridique (qui n'en portait que le nom). Suite à des échanges avec le service administratif de la recherche, nous convînmes finalement que la meilleure option consisterait à m'engager auprès de l'endeuillée à détruire ses données. En vain, je contactai l'enquêtée. À ma connaissance, l'affaire n'avait pas retenti dans le monde funéraire mais ne m'en avait pas moins émotionnellement secouée, tout comme ma collaboratrice stagiaire qui, désœuvrée par la tournure prise par l'entrevue, exprima le besoin d'être protégée et soutenue.

15 Je voulus comprendre et interroger les causes de cette épreuve qui, au-delà du caractère sensible de l'objet (les traces post-mortem), en disait long sur les nécessités d'outiller les pratiques de recherche en dispositifs déontologiques: il fallait non seulement prévenir de possibles écueils méthodologiques mais aussi les émotions des chercheurees. Je savais que dans d'autres contextes comme au Canada, où les SHS 
faisaient l'objet d'un encadrement formel ${ }^{35}$, une telle affaire ne se serait sans doute pas produite. Alors que je publiai dans la revue canadienne Recherches qualitatives pour interroger les méthodes de cette enquête ${ }^{36}$, les retours des évaluations me firent réaliser à quel point la France s'avérait effectivement peu armée sur le plan éthique dans le domaine des SHS et que les chartes y manquaient cruellement. Elles y étaient certes débattues dans certaines instances mais souvent contestées car vues comme un acte de judiciarisation du travail de terrain ${ }^{37}$. Comme la précédente, cette affaire m'invita à explorer les dispositifs d'intégrité scientifique. Je découvris notamment que l'intérêt pour l'éthique et l'intégrité de la recherche par les SHS était récent en France (quand en matière de santé humaine, on s'y intéressait depuis 1999). Les bases, instituées dans le cadre de la charte du CNRS de 2015, avaient été sanctionnées par le rapport Corvol de 2016 qui enfin marquait un tournant ayant pour conséquence la création de l'office Français d'Intégrité Scientifique, en 2017.

16 Constatant les ravages que pouvaient provoquer les déficits de reconnaissance sur le plan humain, je fus progressivement entraînée dans un excès de zèle qui faillit, au nom de la déontologie, me conduire à commettre une faute. Minée par des expériences d'ingratitudes d'universitaires, par des processus d'invisibilisation plus ou moins volontaires ou par le discrédit de "petites mains ", je m'étais fait un point d'honneur à toujours remercier toute contribution «invisible». À l'occasion d'un article sur une enquête de terrain en Arabie saoudite ${ }^{38}$, j'entrepris de remercier une collègue qui m'avait aidée à accéder à son université. Lui demandant de préciser la position et l'affiliation qu'elle souhaitait indiquer, la collègue me spécifia qu'elle refusait d'être citée et que son patronyme ne devrait jamais apparaître. Je frôlai une majestueuse bévue : je n'avais pas tenu compte du contexte politique de l'étude.

Je finirai ce récit sur le constat que ma ténacité pour devenir enseignante-chercheure s'accompagna certes de désillusions mais aussi de gratifications, les premiers sentiments étant paradoxalement au service des seconds. Ce sont en effet mes déceptions et ma crédulité d'un temps qui ont dessillé mon regard et infléchi mon positionnement scientifique. C'est en décelant les enjeux socio-politiques et les stratégies de pouvoir de la science que j'ai pu développer mon sens critique et décrypter autrement les travaux scientifiques. C'est parce que je me sentais exclue du jeu scientifique - peut-être parce que mes origines sociales ne me laissaient prédestiner une telle trajectoire - que je ne m'autorisais pas à la critique. Initialement fascinée par les écrits de Pierre Bourdieu, je me rendis compte que son œuvre travestissait le travail de nombreuses " petites mains » laissées dans l'ombre, voire qu'elle faisait fi - je pense par exemple à La Domination masculine - de travaux féministes majeurs et qu'elle présentait des argumentations orientées ne servant que trop bien la cause du sociologue ${ }^{39}$. C'est également en vertu de ces déconvenues que j'opérai un saut épistémologique dans ma façon d'appréhender le rapport à la science : je m'éloignai des canons de la littérature méthodologique selon lesquels la neutralité dans la recherche s'énonçait comme une règle impérieuse. J'admis que l'objectivité était un mythe et que la revendication des subjectivités pouvait même, comme le montrait l'épistémologie féministe, constituer une force scientifique en ce sens qu'elle favorisait l'engagement et était source de réflexivitéte ${ }^{40}$ En plus de son mérite scientifique, ce positionnement me permettrait de braver d'inévitables jeux de pouvoir qui constituaient autant d'épreuves qui jalonnaient le parcours universitaire et auxquelles on ne pouvait échapper. 


\section{NOTES}

1. Pierre Bourdieu, La noblesse d'État: grandes écoles et esprit de corps, Paris, Minuit, 1989.

2. Pierre Bourdieu, Science de la science et réflexivité. Cours du Collège de France 2000-2001, Paris, Raisons d'agir, 2001.

3. Pierre Bourdieu, La distinction, Paris, Les Éditions de Minuit, 1979.

4. Ibid.

5. Compte tenu des limites de ce texte, j'ai écarté un récit sur les mécanismes qui m'ont conduite à développer des recherches sur le numérique et le genre.

6. Tahar Ben Jelloun, Le racisme expliqué à ma fille, Paris, Seuil, 1998.

7. Gaston Bachelard, La formation de l'esprit scientifique, Paris, J. Vrin, réed.1996 [1 $1^{\text {re }}$ édition : 1938].

8. Gaston Bachelard, op. cit.

9. Pierre Bourdieu, Jean-Claude Chamboredon, Jean-Claude Passeron, Le métier de sociologue, Paris, Mouton, 1983 [1968].

10. Gaston Bachelard, op. cit.

11. Max Weber, Le Savant et le Politique, Préface de Raymond Aron, Traduction de Julien Freund, Bibliothèques 10/18, 2002 [1919].

12. Isabelle Kalinowski, Leçons wébériennes sur la science et la propagande, Marseille, Agone, précédé de M. WEBER, La science, profession et vocation, 2005.

13. Hélène Bourdeloie, «Ressources ouvertes, construction coopérative de la connaissance et fracture numérique. Le cas de l'encyclopédie en ligne Wikipédia ", in Alain Kiyindou (éd), Fractures, mutations, fragmentations : de la diversité des cultures numériques, Paris, Hermès Lavoisier, p. 195-224, 2009.

14. Alan Sokal, «Transgressing the Boundaries. Toward a Transformative Hermeneutics of Quantum Gravity », Social Text, 46-47, 1996, p. 217-252.

15. Bruno Latour, Les microbes. Guerre et paix, suivi de Irréductions, Paris, A.-M. Métaillé, 1984.

16. David Pontille, «L'auteur scientifique en question : pratiques en psychologie et en sciences biomédicales ", Social Science Information, SAGE Publications, 2001, 40(3), pp. 433-453, https:// doi.org/10.1177/053901801040003004. halshs-00261741v2, https://halshs.archives-ouvertes.fr/ halshs-00261741v2/document, consulté le 20 juillet 2020.

17. En vue de respecter la confidentilité des intéressé'e·s, toutes les expériences ici relatées respectent le strict anonymat.

18. David Pontille, 2001, op. cit.

19. Nathalie Heinich, "Invisibilisation de la pensée des femmes", Publictionnaire. Dictionnaire encyclopédique et critique des publics, mis en ligne le 26 février 2020. Accès: http:// publictionnaire.huma-num.fr/notice/invisibilisation-de-la-pensee-des-femmes.

20. Christophe Charle, "L'évaluation des enseignants-chercheurs : Critiques et propositions", Vingtième Siècle. Revue d'histoire, 102(2), p. 159-170, 2009, doi: https://doi.org/10.3917/ving. 102.0159, https://www.cairn.info/revue-vingtieme-siecle-revue-d-histoire-2009-2-page-159.htm, p. 159

21. Devenue depuis l'Hcéres (Haut Conseil de l'évaluation de la recherche et de l'enseignement supérieur).

22. Arnaud Mercier, "Dérives des universités, périls des universitaires », Questions de communication, 22, 2012, p. 197-234.

23. Jean-Richard Cytermann, Pascal Aimé, «La loi "libertés et responsabilités des universités" : origines, apports et bilan de la mise en œuvre ", Revue française d'administration publique, 169(1), 2019, p. 21-36. 
24. David Douyère, Joëlle Le Marec, «Des savoirs vivants de l'enquête à l'écriture de recherche », in Hélène Bourdeloie, David Douyère (éds), Méthodes de recherche sur l'information et la communication, regards croisés, Paris, Mare \& Martin, 2014, p. 117-140.

25. Arnaud Mercier, op. cit.

26. Jean-Noël Darde, «Enseignants-chercheurs, recherche et plagiat », Mouvements, 71(3), 2012, p. 128-137.

27. David Larousserie, « Publier ou périr, une malédiction pour la recherche ». Le Monde Science et techno, 26 septembre 2017: https://tice.agroparistech.fr/coursenligne/courses/ EPISTEMOLOGIEMASTERS/document/

Le_Monde_2017_Publier_ou_perir_une_malediction_pour_la_recherche.pdf?

cidReq=EPISTEMOLOGIEMASTERS, consulté le 20 juin 2020.

28. Laurence Viry, Le monde vécu des universitaires ou la république des égos, Rennes, Presses universitaires de Rennes, 2006.

29. Christophe Charle, 2009, op. cit. p. 170.

30. Arnaud Mercier, op. cit.

31. Charte française de déontologie des métiers de la recherche, janvier 2015 : https://comiteethique.cnrs.fr/wp-content/uploads/

2020/01/2015_Charte_nationale_d\%C3\%A9ontologie_190613.pdf, consulté le 15 juin 2020.

32. Cette pratique est devenue plus systématique avec la circulaire du Secrétaire d'État à l'Enseignement Supérieur et à la Recherche de mars 2017. Voir : https://www.hceres.fr/fr/desacteurs-les-referents-lintegrite-scientifique, consulté le 22 juillet 2020.

33. Laurence Viry, op. cit.

34. Hélène Bourdeloie, «Les impuretés du travail de l'ethnographe sur un terrain sensible. Deuil en ligne et traces numériques des morts ", Recherches qualitatives, 38(2), 2019, p. 25-46.

35. Guillaume Latzko-Toth, Serge Proulx, Enjeux éthiques de la recherche sur le Web, in Christine Barats (éd.), Manuel d'analyse du Web, Paris, Armand Colin, 2013, p. 32-48.

36. Hélène Bourdeloie, 2019, op. cit.

37. Daniel Cefaï, 2003, L'enquête de terrain, Paris, La Découverte.

38. Hélène Bourdeloie, Caterina Gentiloni Silveri, Sara Houmair, « Saudi Women and Socio-Digital Technologies: Reconfiguring Identities », CyberOrient, vol.11, n 1, 2017, http:// www.cyberorient.net/article.do?articleId $=9822$.

39. Nicole-Claude Mathieu, «Bourdieu ou le pouvoir auto-hypnotique de la domination masculine », Les Temps modernes, n 604, 1999, p. 286-324; Jules Falquet, « Pour une anatomie des classes de sexe : Nicole-Claude Mathieu ou la conscience des opprimée·s, Cahiers du Genre, 1(1), 2011, p. 193-217, https://doi.org/10.3917/cdge.050.0193.

40. Bourdeloie, 2019, op. cit.

\section{AUTEUR}

\section{HÉLÈNE BOURDELOIE}

Université Sorbonne Paris Nord, LabSIC \& chercheure associée au CARISM (Université Paris II Panthéon-Assas) 